\title{
Mask roughness induced LER control and mitigation: aberrations sensitivity study and alternate illumination scheme
} Brittany M. McClinton ${ }^{1}$ and Patrick P. Naulleau ${ }^{2}$

\author{
${ }^{1}$ Dept. of Electrical Engineering, University of California, Berkeley, CA 94720 \\ ${ }^{2}$ Center for X-Ray Optics, Lawrence Berkeley National Laboratory, Berkeley, CA 94720
}

\begin{abstract}
Here we conduct a mask-roughness-induced line-edge-roughness (LER) aberrations sensitivity study both as a random distribution amongst the first 16 Fringe Zernikes (for overall aberration levels of $0.25,0.50$, and $0.75 \mathrm{~nm}$ rms) as well as an individual aberrations sensitivity matrix over the first 37 Fringe Zernikes. Full 2D aerial image modeling for an imaging system with NA $=0.32$ was done for both the 22-nm and $16-\mathrm{nm}$ half-pitch nodes on a rough mask with a replicated surface roughness (RSR) of $100 \mathrm{pm}$ and a correlation length of $32 \mathrm{~nm}$ at the nominal extreme-ultraviolet lithography (EUVL) wavelength of $13.5 \mathrm{~nm}$. As the ideal RSR value for commercialization of EUVL is $50 \mathrm{pm}$ and under, and furthermore as has been shown elsewhere, a correlation length of $32 \mathrm{~nm}$ of roughness on the mask sits on the peak LER value for an NA $=0.32$ imaging optic, these mask roughness values and consequently the aberration sensitivity study presented here, represent a worst-case scenario. The illumination conditions were chosen based on the possible candidates for the $22-\mathrm{nm}$ and $16-\mathrm{nm}$ half-pitch nodes, respectively. In the $22-\mathrm{nm}$ case, a disk illumination setting of $\sigma=0.50$ was used, and for the $16-\mathrm{nm}$ case, crosspole illumination with $\sigma=0.10$ at an optimum offset of $\mathrm{dx}=0$ and $\mathrm{dy}=.67$ in sigma space. In examining how to mitigate mask roughness induced LER, we considered an alternate illumination scheme whereby a traditional dipole's angular spectrum is extended in the direction parallel to the line-andspace mask absorber pattern to represent a "strip". While this illumination surprisingly provides minimal improvement to the LER as compared to several alternate illumination schemes, the overall imaging quality in terms of image-logslope (ILS) and contrast is improved.
\end{abstract}

Keywords: extreme ultraviolet, lithography, aberration, Zernike, line-edge-roughness, mask roughness, speckle

\section{INTRODUCTION}

As extreme-ultraviolet lithography (EUVL) moves towards commercialization, meeting the benchmark for line-edge roughness (LER) remains a significant challenge. As stated by the International Roadmap for Semiconductors [1], LER should be kept to less than $1.2 \mathrm{~nm}$ for the 22-nm half pitch node; current state-of-the-art extreme-ultraviolet lithography (EUVL) tools are only capable of consistently achieving roughly $3 \mathrm{~nm}$ LER. It is apparent that improvements need to be made in understanding the principle causes of LER. At EUV wavelengths, there are three main contributors: LER induced by the resist, LER inherently present on the mask absorber pattern, and mask roughness induced LER. Much research has already been done on the first two factors- resist LER and mask LER [2,3]. Recent progress, too, has been made in understanding the extent of mask roughness induced LER [4], but only in the case of ideal optics. This work is an extension of that effort to understand the nature of mask roughness induced LER in the presence of optical aberrations.

As previously recorded in the literature [5-7], mask surface roughness on a reflective mask geometrically encodes a phase roughness on the wavefront that, due to the reflection, is a factor of two larger than the actual surface deviations. For EUVL, the consequences are particularly detrimental considering the nominal illumination wavelength of $13.5 \mathrm{~nm}$; very small roughnesses on the multi-layer mask significantly modulate the phase. Furthermore, as in all projection lithography techniques, the process itself is band-limited, resulting in imperfect replication of the mask object that is sensitive to the phase errors on it. The phase roughness induced by the mask surface results in many overlapping amplitude spread function in the image plane, all differing in phase from one to another. This causes a complex interference pattern phenomenon, known as speckle, which is modulated by the coherence factor. Speckle, which is essentially small intensity deviations, induces in turn small variations in line-edge position of the features we wish to 
print by virtue of thresholding in the lithography process. The sensitivity of LER to speckle is determined by the imagelog-slope (ILS). A smaller ILS indicates larger line-edge movements for a given increment of intensity variation, e.g., speckle. In the presence of aberrations of the imaging optic, the imaging quality is degraded, as manifest in a smaller ILS. For instance, in considering defocus as an aberration, it is well-known that the ILS worsens as more aberration (defocus) is introduced into the system, and consequently, the susceptibility to speckle increases accordingly. We present here a full aberration sensitivity study on the mask roughness induced LER for the first 37 Fringe Zernikes conducted with fully simulated 2D aerial image modeling. This was done for both 22-nm and 16-nm lines and spaces using potential illumination conditions for each of those nodes respectively, with an NA of 0.32. Mask roughness conditions were set at a replicated surface roughness (RSR) value of $100 \mathrm{pm}$, a correlation length of $32 \mathrm{~nm}$, and a roughness exponent of infinity [4]. Since the ideal RSR specification for commercialization of EUVL at the 22-nm node is less than $50 \mathrm{pm}$, and furthermore, since it has been shown that a $32 \mathrm{~nm}$ correlation length sits directly on the peak of mask roughness induced LER (determined by the objective NA) [4], these results represent a worst-case scenario. The modeling for the 22-nm features are presented in section 2, and that for 16-nm is presented in section 3.

We also consider an alternative illumination scheme specifically aimed at mitigating mask roughness induced LER. In this scheme, a traditional dipole angular spectrum is extended in the direction parallel to the line-and-space mask absorber pattern to represent a "strip". Modeling and results are presented in section 4.

\section{ABERRATIONS AT THE 22-NM NODE}

Following the numerical analysis of mask roughness induced LER described elsewhere [7], we randomly generated a mask object that was $1024 \times 1024$ pixels at $1 \mathrm{~nm} /$ pixel with 1:1 imaging to the wafer plane. This statistical representation of a clear rough mask was then appropriately scaled so that the standard deviation of this height map matched the desired RSR value of $100 \mathrm{pm}$ and a correlation length of $32 \mathrm{~nm}$. The process was repeated to generate an ensemble of 10 random realizations of the clear rough mask, all with the desired RSR and correlation length values. Given the nominal illumination wavelength of $13.5 \mathrm{~nm}$ for EUVL, we converted each topographic surface to a phase perturbation, determined by from the geometric path length differences imparted by the rough surface of the mask. Finally, we overlaid (multiplied) each clear-field rough mask realization with binary $22 \mathrm{~nm}$ lines and spaces.

Afterwards, we conducted full 2D aerial image simulations using in-house software based on the equations of partial coherence image formulation [8]. Commercial software with similar capabilities include PROLITH [9] and Panoramic [10]. This was done for disk illumination with a partial coherence factor $\sigma=0.50$, first for an aberration-free optical system with NA $=0.32$ from through focus from $-90 \mathrm{~nm}$ to $+90 \mathrm{~nm}$. The low NA allows for scalar and thin mask modeling. Following simulations, we extracted the LER in the offline analysis package, SUMMIT [11], after appropriately sizing features to target $\mathrm{CD}$ at best focus. These results are presented in Figure 1. The results indicate that one single statistical realization of the rough mask well represents the entire ensemble for an ideal pupil map.



Fig. 1. 10 random rough mask realizations all with RSR $100 \mathrm{pm}$, correlation length $32 \mathrm{~nm}$, on an ideal pupil map of NA $=0.32$ with disk illumination of $\sigma=0.50$ for $22 \mathrm{~nm}$ lines and spaces. 
Next we repeat the simulations, now introducing aberrations into the system. We randomly generated a distribution in Fringe Zernikes 5 through 16 (ignoring the first four: piston, x-tilt,y-tilt, and power), and scaled to $0.25 \mathrm{~nm} \mathrm{rms}$ wavefront deviation, or 18.5 milliwaves. This was repeated to generate an ensemble of 10 random realizations all with $0.25 \mathrm{~nm} \mathrm{rms}$ wavefront deviation for Fringe Zernikes 5 through 16. This same distribution amongst Fringe Zernikes 516 was scaled up for two higher levels of aberrations, one at $0.50 \mathrm{~nm}$ rms wavefront deviation (37.0 milliwaves) and also at $0.75 \mathrm{~nm}$ rms wavefront deviation (55.6 milliwaves). Initially, we consider the variation of just one random realization of the Zernike distribution against all 10 random realizations of the rough mask. The simulation results for mask roughness induced LER through focus are presented in Figure 2. The results indicate that just one realization of the mask roughness well represents the entire ensemble at all levels of aberrations. Using, therefore, just one realization of the mask roughness, we next consider the variations in mask roughness induced LER by the previously generated 10 random realizations of the Zernike distribution in Fringe Zernikes 5-16. Those results are seen in Figure 3. We see that as the amount of aberrations increase from $0.25 \mathrm{~nm}$ to $0.50 \mathrm{~nm}$ to $0.75 \mathrm{~nm}$ total wavefront deviations, the consistency of one random realization of the Zernike distribution to another gradually decreases. At the $0.25 \mathrm{~nm}$ wavefront deviation level, the ensemble seems internally consistent even at large defocus, to within $1 \mathrm{~nm}$ LER of each other. At the $0.75 \mathrm{~nm}$ wavefront deviation level, individual realizations of the ensemble may deviate by as much as $3.5 \mathrm{~nm}$ LER from each other. This wide variation in resultant LER levels indicates that the system is highly sensitive to particular Fringe Zernike aberrations.

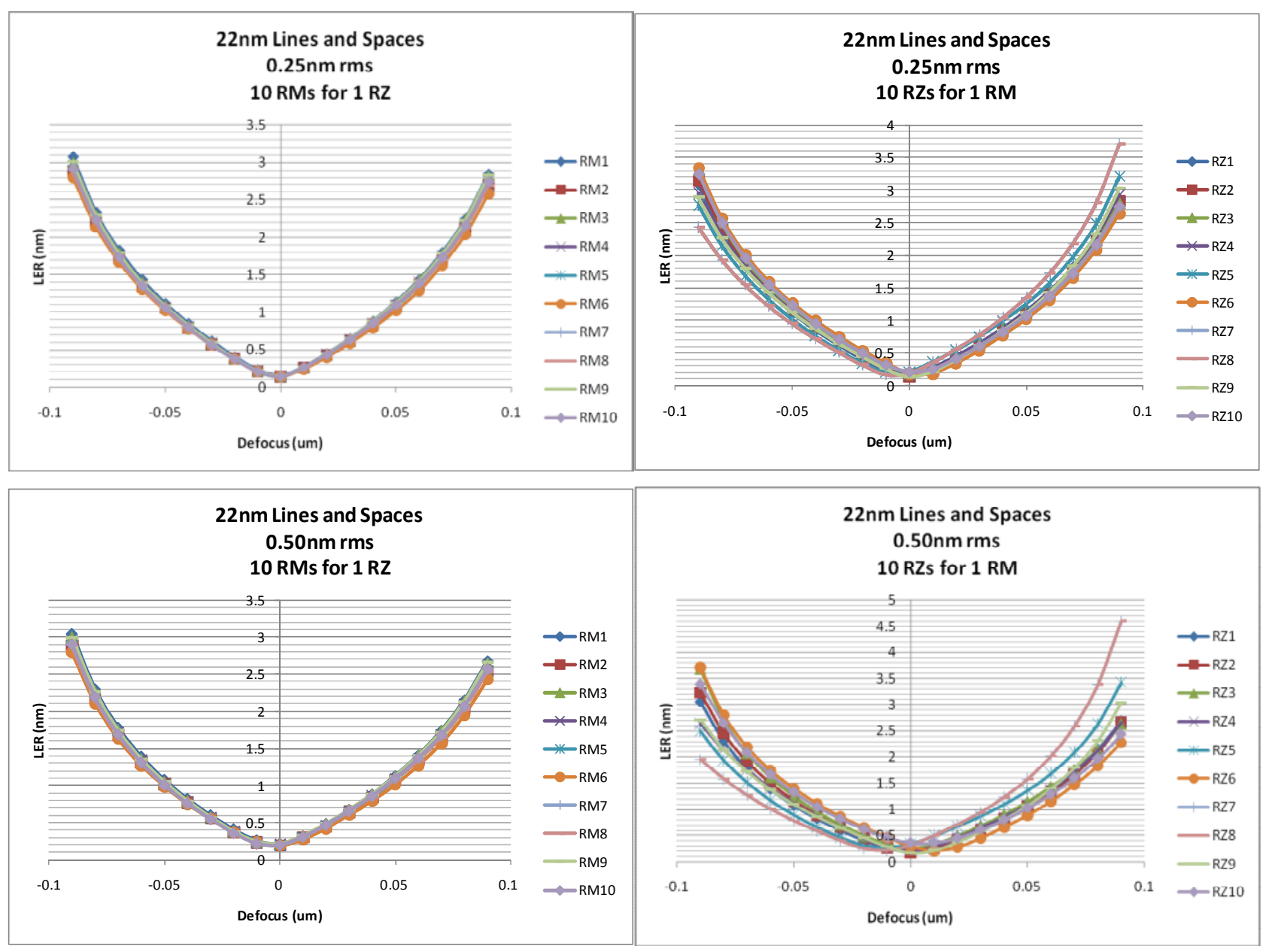




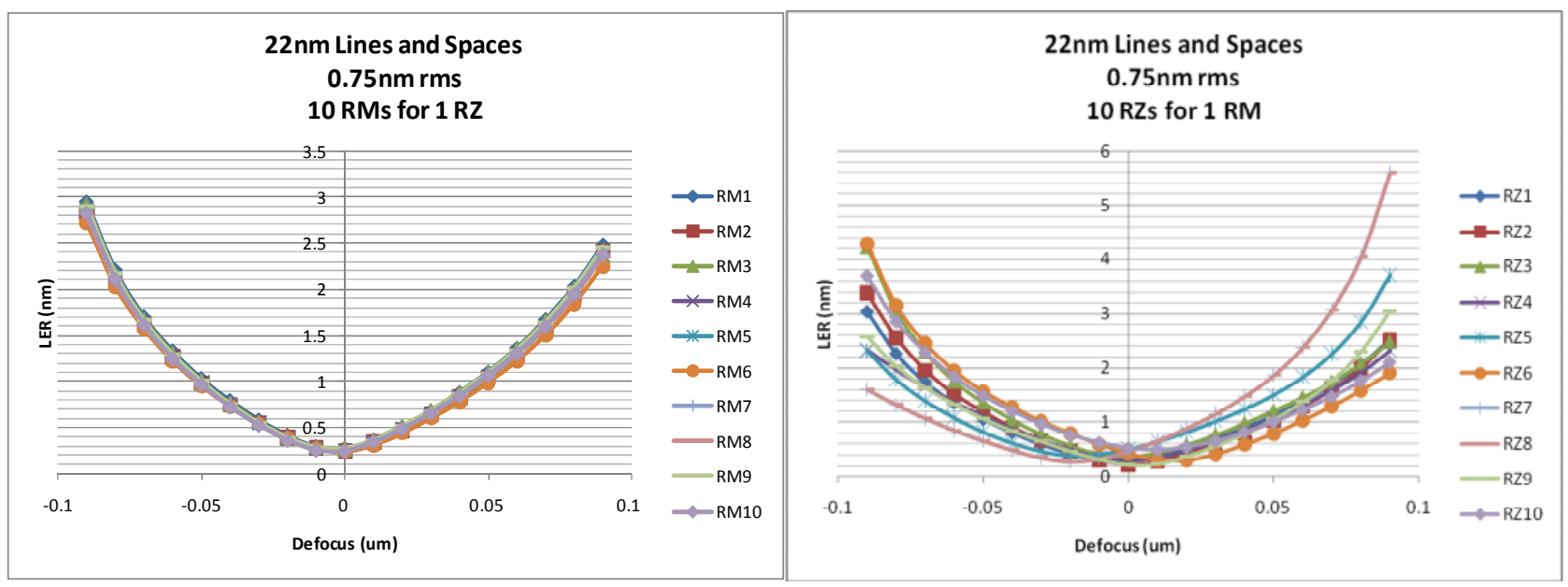

Fig. 2. (left column) 10 random rough mask realizations (RMs) all with RSR 100pm, correlation length 32nm, on 1 random Zernike distribution (RZ) amongst fringe zernikes 5-16 for total aberrations levels of $0.25,0.50$, and $0.75 \mathrm{~nm}$ rms, with an NA = 0.32 with disk illumination of $\sigma=0.50$ for $22 \mathrm{~nm}$ lines and spaces.

Fig. 3. (right column) 1 random rough mask realization (RM) all with RSR 100pm, correlation length $32 \mathrm{~nm}$, on 10 random Zernike distributions (RZs) amongst fringe zernikes 5-16 for total aberrations levels of $0.25,0.50$, and $0.75 \mathrm{~nm}$ rms, with an of NA $=0.32$ with disk illumination of $\sigma=0.50$ for $22 \mathrm{~nm}$ lines and spaces.

Despite the significant variations between each individual realization of the Zernike distribution, we averaged the 10 realizations for each level of aberrations and plotted them against one another (see Figure 4). These results indicate as a general trend, improving the level of total aberrations in the imaging system has a minimal effect on the mask roughness induced LER, except for a noticeable difference at best focus.

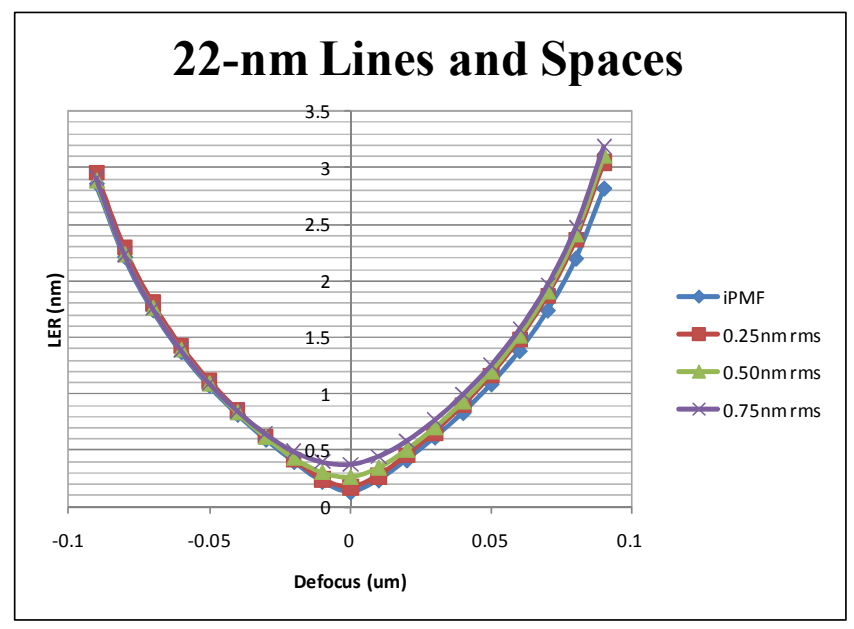

Fig. 4. 1 random rough mask realization $(\mathrm{RM})$ all with RSR 100pm, correlation length 32nm, averaged against 10 random Zernike distributions (RZs) amongst fringe zernikes 5-16 for total aberrations levels of $0.25,0.50$, and $0.75 \mathrm{~nm}$ rms, with NA $=0.32$ with disk illumination of $\sigma=0.50$. For "iPMF", this indicates the average of the $10 \mathrm{RMs}$ on an ideal pupil map graphed previously in Figure 1 .

To investigate this further, we conducted a sensitivity matrix study of Fringe Zernikes 5 through 37 . For instance, in looking at Fringe Zernike 5, we concentrated all aberrations within this one Zernike, and modeled on just one realization of mask roughness. We did this for three levels of aberrations $(0.25 \mathrm{~nm}, 0.50 \mathrm{~nm}$, and $0.75 \mathrm{~nm}$ rms wavefront deviations), again for Zernikes 5 through 37. Those results can be seen in Figure 5. At the $0.25 \mathrm{~nm}$ rms wavefront deviation level, we see that the process is in particular sensitive to Fringe Zernikes 5 ( $3^{\text {rd }}$ order astigmatism) and 8 ( $3^{\text {rd }}$ order spherical), and to some extent 25 ( $7^{\text {th }}$ order spherical). The sensitivity to these particular Fringe Zernikes remain consistent at the higher levels of aberrations $(0.50 \mathrm{~nm}$ and $0.75 \mathrm{~nm}$ wavefront deviations). We note that in the case of each other Fringe Zernike, there is not much variation, even amongst the different aberration levels: all converge to 
approximately the $2.5 \mathrm{~nm}$ to $3 \mathrm{~nm}$ LER range at extreme defocus values, no matter if the wavefront deviations are at the $0.25 \mathrm{~nm}$ rms level or the $0.75 \mathrm{~nm}$ rms level. This is consistent with Fig. 4, where, again, improvements in total wavefront deviations across the distribution of Fringe Zernikes 5-16 minimally affected the mask roughness induced LER. Efforts should rather be made in improving the mask surface roughness specifications such as RSR and correlation length, which as shown previously [4], can have a much more significant or atleast comparable impact. However, in practice, it might be difficult to significantly improve the RSR beyond $100 \mathrm{pm}$.

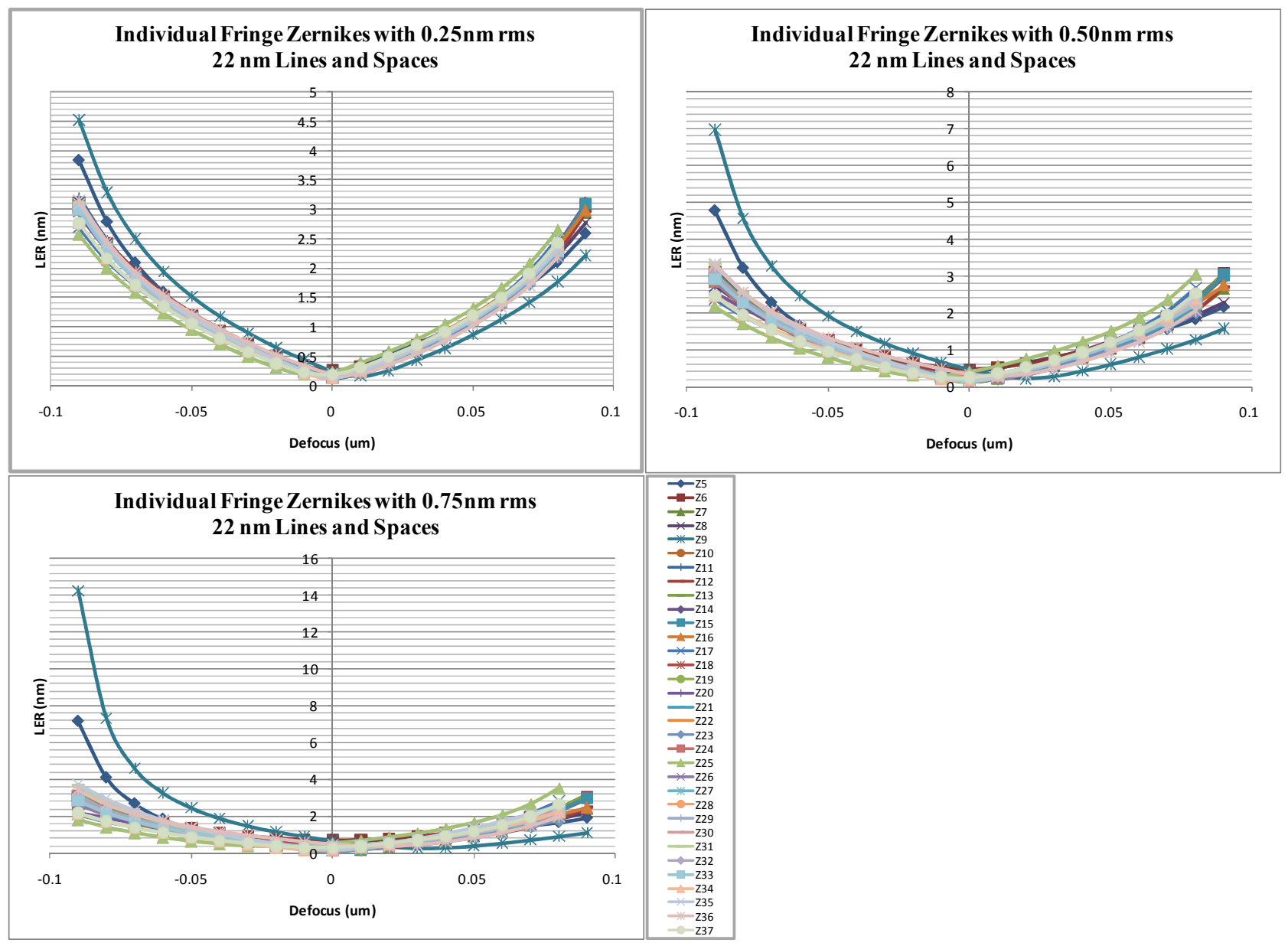

Fig. 5. 1 random rough mask realization (RM) all with RSR 100pm, correlation length 32nm, on each individual fringe zernike 5-37 for a concentrated aberration level in that fringe zernike of $0.25,0.50$, and $0.75 \mathrm{~nm} \mathrm{rms}$, with an of NA $=0.32$ with disk illumination of $\sigma=0.50$ for $22 \mathrm{~nm}$ lines and spaces.

\section{ABERRATIONS AT THE 16-NM NODE}

We repeat the entire analysis above now for 16-nm lines and spaces case, again with an NA $=0.32$, RSR of $100 \mathrm{pm}$, and a correlation length of $32 \mathrm{~nm}$. The only difference is a change in illumination conditions, now set to crosspole illumination with $\sigma=0.10$ and an offset of $\mathrm{dx}=0$ and $\mathrm{dy}=.67$ in sigma space.

Following the analysis process above, we started with simulating an aberration-free optical system with NA $=0.32$ again for a focal range of $-90 \mathrm{~nm}$ to $+90 \mathrm{~nm}$. After appropriately sizing features to target $C D$ at best focus, the results are 
presented in Figure 6 and show that once again each statistical realization of the rough mask well represents the entire ensemble for an ideal pupil map.

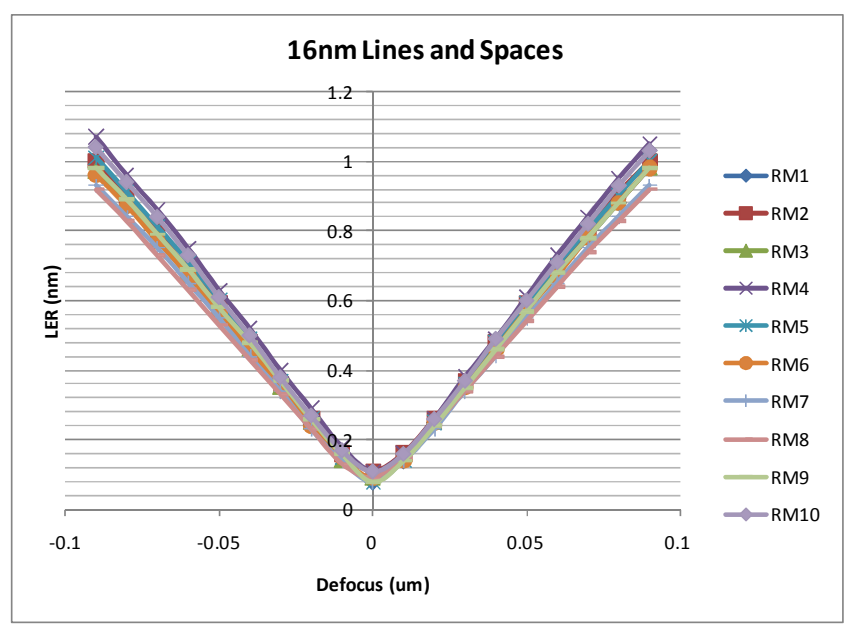

Fig. 6. 10 random rough mask realizations all with RSR $100 \mathrm{pm}$, correlation length $32 \mathrm{~nm}$, on an ideal pupil map of NA $=0.32$ with crosspole illumination of $\sigma=0.10$ optimized for $16 \mathrm{~nm}$ lines and spaces.

Now introducing aberrations into the system, we once more consider the variation of just one random realization of the Zernike distribution over Fringe Zernikes 5 through 16, against all 10 random realizations of the rough mask (for overall aberration levels of $0.25,0.50$, and $0.75 \mathrm{~nm} \mathrm{rms}$ ). The results similarly indicate that just one realization of the mask roughness well represents the entire ensemble at all levels of aberrations (see Fig. 7). We therefore continue with our previous simplification, and use just one realization of the mask roughness for the rest of our analysis. As this single mask roughness realization is sampled across the same previously generated 10 random realizations of the Zernike distribution in Fringe Zernikes 5-16, the dependency of LER on which realization of the Zernike distribution remains quite small at the $0.25 \mathrm{~nm}$ rms level, with substantial deviations ( $\sim 0.2 \mathrm{~nm}$ LER) between realizations only observed at best focus (see Fig. 8). As overall aberrations increase to the 0.50 and $0.75 \mathrm{~nm}$ level, these deviations become quite large at best focus, as much as $0.8 \mathrm{~nm}$ LER increase at zero defocus, while the LER remains pinned to about 1-1.2 $\mathrm{nm}$ LER at the extremes of focus (again, see Figure 8). From this, we conclude that the system is still highly sensitive to particular fringe Zernike aberrations.

Once more, we conduct an averaging analysis of the 10 random realizations of the Zernike distribution, despite the significant variations in system LER performance between each realization. In contrast to the $22 \mathrm{~nm}$ lines and spaces case, at the $16 \mathrm{~nm}$ node, LER is significantly more sensitive to the overall aberrations level throughout focus. Aberration levels of $0.25 \mathrm{~nm}$ rms could perhaps be tolerated, which already sacrifice $0.3 \mathrm{~nm}$ LER at best focus (see Figure 9).

Finally, the sensitivity matrix study of Fringe Zernikes 5 through 37 revealed a particular sensitivity to Fringe Zernikes 8 and $24\left(3^{\text {rd }}\right.$ and $7^{\text {th }}$ order spherical), as well as $27\left(5^{\text {th }}\right.$ order quadrafoil) at the $0.25 \mathrm{~nm}$ rms level (see Figure 10$)$. These particular aberrations remain dominant at higher aberration levels as well.

We note that the results above are extremely dependent not only on the nominal feature sizes of 16 and $22 \mathrm{~nm}$, but also the type of illumination (crosspole $\sigma=0.10$ and disk $\sigma=0.50$ respectively, in this case). The overall levels of LER should improve accordingly with a significant reduction in the RSR value, and/or shift in the correlation length. 




Fig. 7. (left column) 10 random rough mask realizations (RMs) all with RSR 100pm, correlation length $32 \mathrm{~nm}$, on 1 random Zernike distribution (RZ) amongst fringe zernikes 5-16 for total aberrations levels of $0.25,0.50$, and $0.75 \mathrm{~nm}$ rms, with crosspole illumination of $\sigma=0.10$ optimized for $16 \mathrm{~nm}$ lines and spaces.

Fig. 8. (right column) 1 random rough mask realization (RM) all with RSR 100pm, correlation length $32 \mathrm{~nm}$, on 10 random Zernike distributions (RZs) amongst fringe zernikes $5-16$ for total aberrations levels of $0.25,0.50$, and $0.75 \mathrm{~nm}$ rms, with an of NA $=0.32$ with crosspole illumination of $\sigma=0.10$ optimized for $16 \mathrm{~nm}$ lines and spaces. 


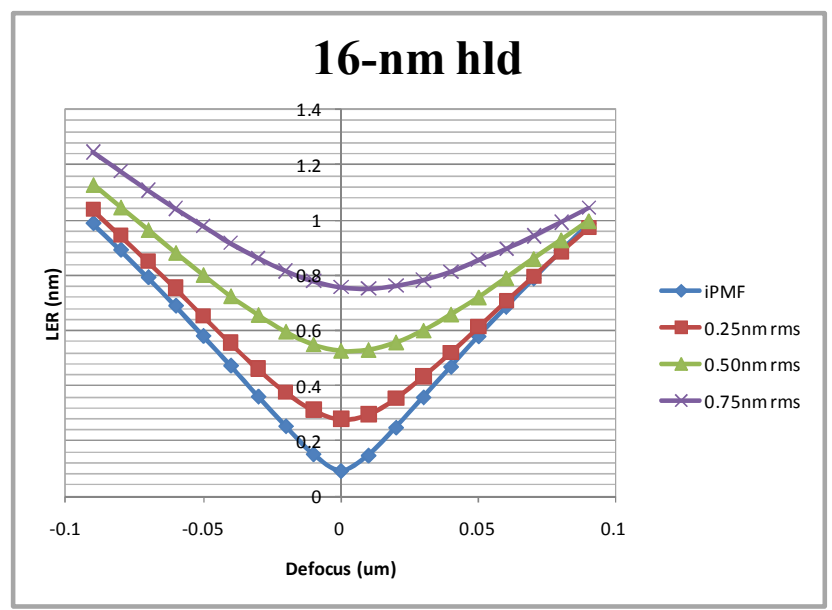

Fig. 9. 1 random rough mask realization $(\mathrm{RM})$ all with RSR $100 \mathrm{pm}$, correlation length $32 \mathrm{~nm}$, averaged against 10 random Zernike distributions (RZs) amongst fringe zernikes 5-16 for total aberrations levels of $0.25,0.50$, and $0.75 \mathrm{~nm}$ rms, with NA $=0.32$ with crosspole illumination of $\sigma=0.10$ optimized for $16 \mathrm{~nm}$ lines and spaces. For "iPMF", this indicates the average of the 10 RMs on an ideal pupil map graphed previously in Figure 6.

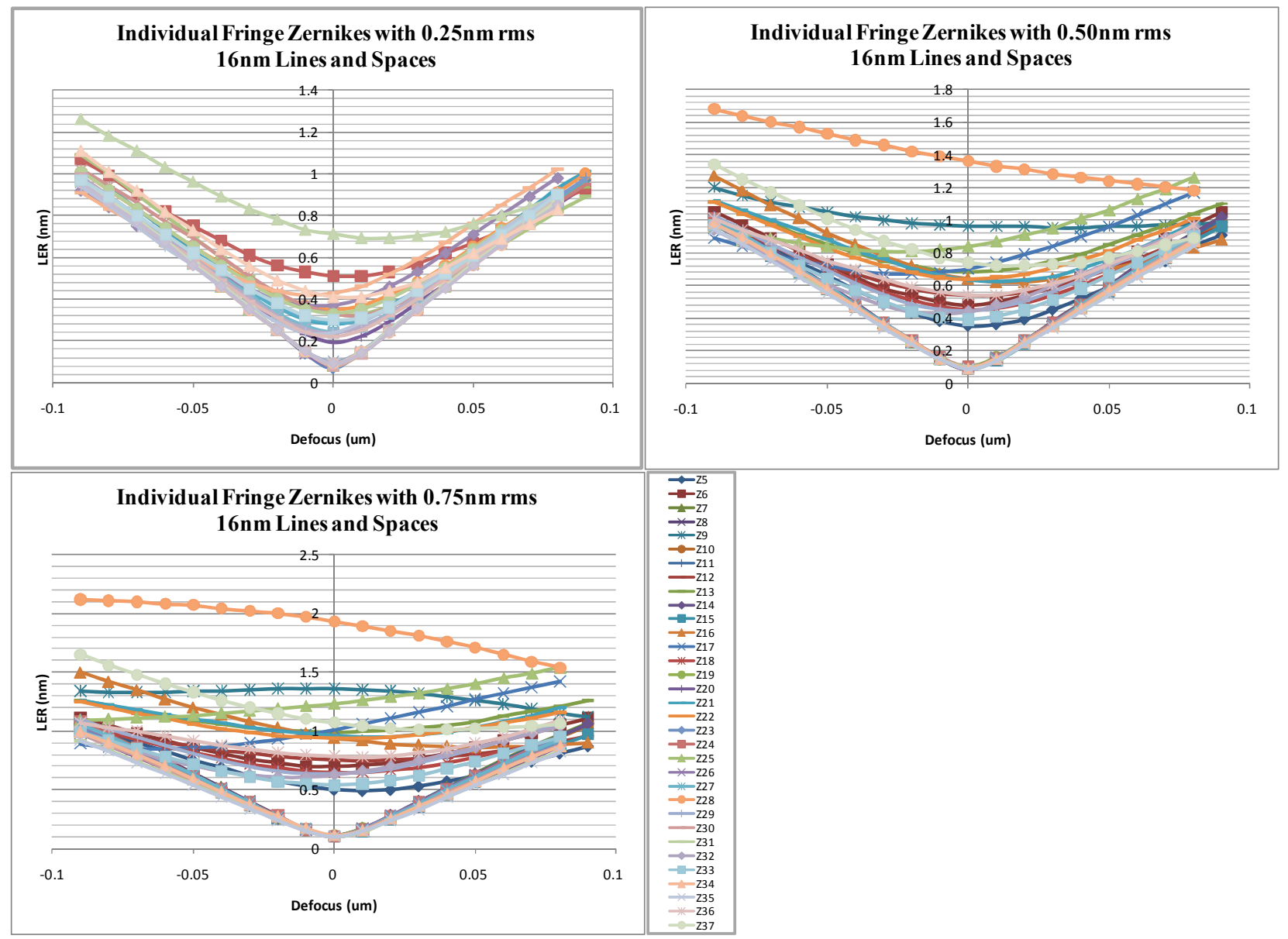

Fig. 10. 1 random rough mask realization (RM) all with RSR 100pm, correlation length 32nm, on each individual fringe zernike 5-37 for a concentrated aberration level in that fringe zernike of $0.25,0.50$, and $0.75 \mathrm{~nm}$ rms, with an of NA $=0.32$ with crosspole illumination of $\sigma=0.10$ optimized for $16 \mathrm{~nm}$ lines and spaces. 


\section{EXTENDED DIPOLE "STRIP" ILLUMINATION}

Recent studies have shown the explicit relationship between speckle (and hence source coherence), aerial image (through image-log-slope, ILS), and LER [4]. From this basic relationship, we can conclude that if speckle is reduced while maintaining good ILS, indicative of good imaging quality, LER should be mitigated. Once such way we can envision doing this is by an alternate illumination scheme whereby a traditional dipole angular spectrum is extended in the direction parallel to the line-and-space mask absorber pattern to represent a "strip". We hypothesized that this reduction in coherence in direction parallel to the predominant pattern orientation should effectively reduce the speckle arising from mask roughness, while the high coherence in the orthogonal direction should maintain good resolution and high ILS.

We conducted our analysis by gradually extending the dipole from an initial $\sigma=0.20$ radius (properly displaced for 16 $\mathrm{nm}$ lines and spaces at $\mathrm{dx}=0$ and $\mathrm{dy}=.67$ in sigma space) in four gradual steps, each time increasing the length of the dipole by $\Delta \mathrm{x}=0.20$ in sigma space to either side of the dipole (see Figure 10). For each illumination condition, we modeled the system on an aberration-free $\mathrm{NA}=0.32 \mathrm{optic}, 13.5-\mathrm{nm}$ illumination wavelength, and the same 10 random realizations ensemble of mask roughness for RSR $100 \mathrm{pm}$ and correlation length of $32 \mathrm{~nm}$ used above.
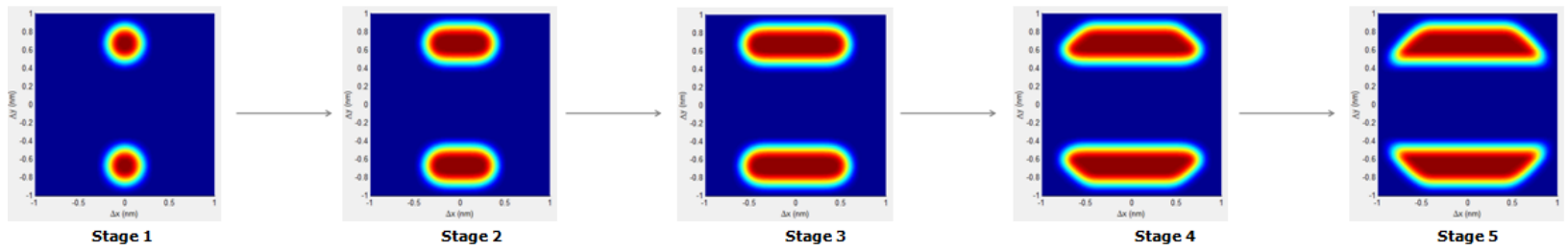

Fig. 10. The five stages of extended dipole illumination tested for LER sensitivity, starting with dipole illumination with intrinsic $\sigma=$ 0.20 optimized for $16 \mathrm{~nm}$ lines and spaces.

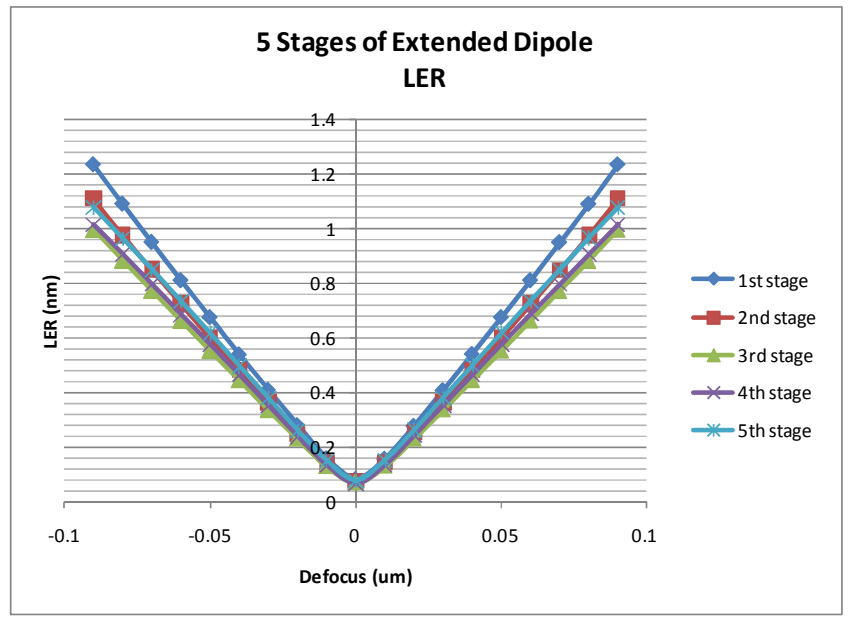

Fig. 11. The LER performance of the five stages of extended dipole.

Show in Figure 11 are the five stages of the extended dipole LER results plotted through focus. They show that the extension of the dipole gradually benefits the LER (by about $0.2 \mathrm{~nm}$ LER at the extremes of focus) until the point that the dipole begins to be clipped by the pupil (after stage 3 extended dipole). Compared directly to LER results of the crosspole illumination examined previously in section 3, this illumination surprisingly provides nearly the same LER values (see Figure 12). We suspect that this results because the LER seems to be governed by the so-called "geometric" regime, a concept being explored simultaneously by this research group [12]. Although there is little LER improvement with the extended dipole compared to the crosspole illumination scheme, a quick look at the imaging quality in terms of the tradition ILS and contrast (Figure 13) show that the extended dipole stage 3 offers potentially improved performance. Not to mention, the larger absolute source size may increase throughput at the wafer level. The surprisingly minimal improvement in LER with the extended dipole runs counter-intuitively to our initial hypothesis. 


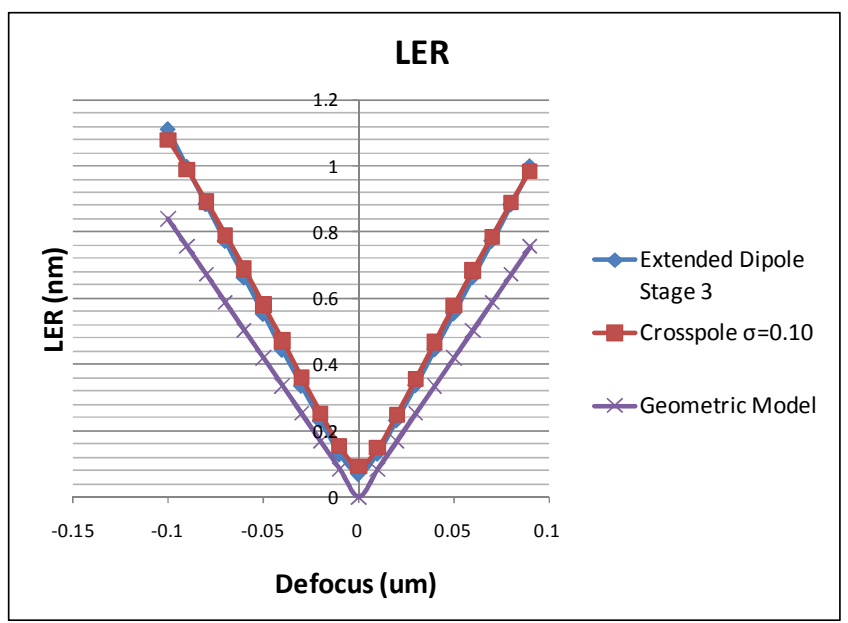

Fig. 12. LER comparison between the optimal $3^{\text {rd }}$ stage of extended dipole, versus crosspole illumination of $\sigma=0.10$ optimized for $16 \mathrm{~nm}$ lines and spaces, and the geometric model.

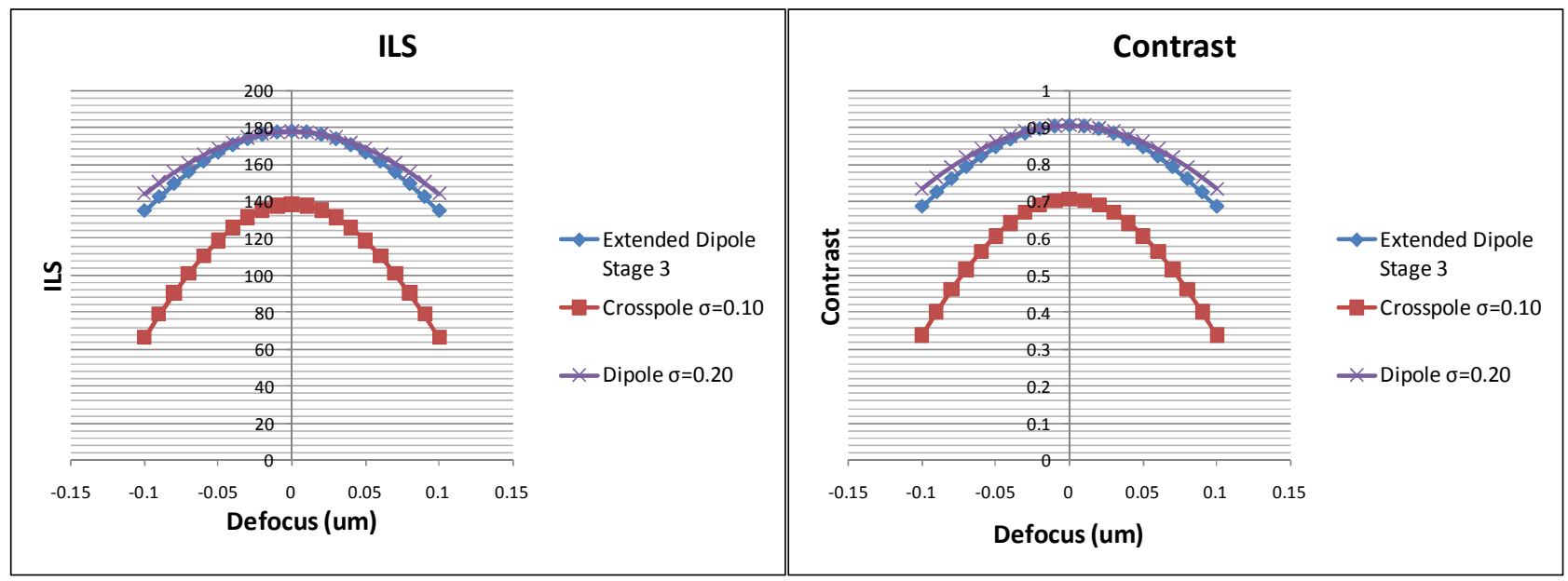

Fig. 13. ILS and Contrast comparison between the optimal $3^{\text {rd }}$ stage of extended dipole, versus crosspole illumination of $\sigma=0.10$, and the original dipole ( $1^{\text {st }}$ stage of extended dipole) of $\sigma=0.20$, all optimized for $16 \mathrm{~nm}$ lines and spaces.

\section{CONCLUSIONS}

Our examinations of $22 \mathrm{~nm}$ lines and spaces under disk illumination of $\sigma=0.50$ and NA $=0.32$ show that aberrations between $0.25-0.75 \mathrm{~nm}$ rms levels yield similar LER performance through focus. Efforts would be better spent on improving mask roughness characteristics. The individual Fringe Zernike study show a particular sensitivity to 5 (3rd order astigmatism) and 8 (3rd order spherical), and to some extent 25 (7th order spherical).

For 16-nm lines and spaces under optimized crosspole illumination of $\sigma=0.10$ and NA $=0.32$, overall aberrations levels should be limited to $0.25 \mathrm{~nm}$ rms. Fringe Zernikes 8 and 24 ( $3 \mathrm{rd}$ and 7 th order spherical), as well as 27 (5th order quadrafoil) are particularly sensitive. As with the 22-nm node, aberration improvements should be made hand in hand with mask roughness improvements, as both are equally important from an LER perspective. Of course, all of the results above are extremely dependent not only on the nominal feature sizes of 16 and $22 \mathrm{~nm}$, but also the type of illumination used. 
The extended dipole illumination stage 3 (intrinsically $\sigma=0.20$ ) explored for possible use at the 16 -nm node may provide an LER, ILS, and contrast improvement over crosspole illumination $(\sigma=0.10)$, and greater throughput than traditional dipole illumination.

The authors are grateful for the support of this work by the National Science Foundation EUV Science and Technology Center. The work was performed at Lawrence Berkeley National Laboratory's Advanced Light Source synchrotron facility and was supported in part by SEMATECH through the U.S. Department of Energy under Contract No. DEAC02-05CH11231.

\section{REFERENCES}

1. International Technology Roadmap for Semiconductors, 2009 ed., http://public.itrs.net/.

2. G. Gallatin, "Resist Blur and Line Edge Roughness," Proc. SPIE 5754, 38-52 (2005).

3. P. Naulleau and G. Gallatin, "Line-edge roughness transfer function and its application to determining mask effects in EUV resist characterization," Appl. Opt. 42, 3390-3397 (2003).

4. B. McClinton and P. Naulleau, "Mask roughness induced LER: a rule-of-thumb," J. Micro/Nanolith. MEMS and MOEMS 9, 041208 (2010).

5. T. Milster and N. Beaudry, "Scattering and Coherence in EUVL," in Emerging Lithographic Technologies II, Y. Vladimirsky, ed., Proc. SPIE 3331, 537-543 (1998).

6. P. Naulleau and S. George, "Implications of image plane line-edge roughness requirements on extreme ultraviolet mask specifications," Proc. SPIE 7379, 1-11 (2009).

7. P. Naulleau, "Relevance of mask-roughness-induced printed line-edge roughness in recent and future extremeultraviolet lithography tests," Appl. Opt. 43, 4025-4032 (2004).

8. J. W. Goodman, Statistical Optics (Wiley, New York, 1985), Chap. 7, pp. 286-360.

9. PROLITH is a registered trademark of KLA-Tencor Corporation, 160 Rio Robles, San Jose, Calif. 95134.

10. Panoramic Technology, Inc., "Em-suite: core lithography simulation package,” (2009), see http://www.panoramictech.com.

11. SuMMIT Software Division of EUV Technology, SUMMIT Litho Image Analysis Software, 2009, http://www.euvl.com/summit/.

12. B. McClinton and P. Naulleau, "Mask roughness induced LER: geometric model at long correlation lengths," Proc. SPIE, to be published (2011). 


\section{DISCLAIMER}

This document was prepared as an account of work sponsored by the United States Government. While this document is believed to contain correct information, neither the United States Government nor any agency thereof, nor The Regents of the University of California, nor any of their employees, makes any warranty, express or implied, or assumes any legal responsibility for the accuracy, completeness, or usefulness of any information, apparatus, product, or process disclosed, or represents that its use would not infringe privately owned rights. Reference herein to any specific commercial product, process, or service by its trade name, trademark, manufacturer, or otherwise, does not necessarily constitute or imply its endorsement, recommendation, or favoring by the United States Government or any agency thereof, or The Regents of the University of California. The views and opinions of authors expressed herein do not necessarily state or reflect those of the United States Government or any agency thereof or The Regents of the University of California. 